\title{
Phenotypic Antimicrobial Resistance Patterns in Salmonella typhimurium and Enteritidis Strains Isolated from Human, Food, and Environmental Samples of Broiler Meat Production Chain in Punjab
}

\author{
Shakera Sadiq ${ }^{1 *}$, Mansur-ud-Din Ahmad ${ }^{1}$, Mamoona Chaudhry ${ }^{1}$, Haroon Akbar ${ }^{2}$, Muhammad Hassan Mushtaq ${ }^{1}$, \\ Junaid Sadiq ${ }^{\mathbf{3}}$ and Saima Hasan ${ }^{\mathbf{1}}$ \\ ${ }^{1}$ Department of Epidemiology and Public Health, University of Veterinary and Animal Sciences, Lahore 54000, Pakistan \\ ${ }^{2}$ Department of Parasitology, University of Veterinary and Animal Sciences, Lahore 54000, Pakistan \\ ${ }^{3}$ Department of Environmental Sciences, Quaid-i-Azam University, Islamabad 44000, Pakistan \\ *For correspondence: shakera.sadiq@uvas.edu.pk \\ Received 17 February 2021; Accepted 24 May 2021; Published 10 July 2021
}

\begin{abstract}
The emergence of antimicrobial resistance is a growing public health issue worldwide due to extensive use in agriculture, food, and veterinary medicine. The rate of antimicrobial resistance varies with different antibiotics and serotypes but Salmonella enterica serovar Enteritidis is one of the most widespread serotypes which is comparatively more susceptible to antimicrobial resistance followed by S. Typhimurium. A total of 71 Salmonella strains (S. Typhimurium, n=45; S. Enteritidis, $\mathrm{n}=26$ ) isolated from humans, food and the environment were used in the study. Both isolates were confirmed through PCR by targeting their specific spy and $s d f$ genes respectively. Kirby-Bauer disc diffusion method was used to determine antimicrobial susceptibility against 10 antibiotics used. An exponentially high level of resistance was found in S. Typhimurium strains. The highest level of resistance was found against amoxicillin (97.78\%) followed by tetracycline (95.56\%), gentamicin (93.33\%), trimethoprim $(86.67 \%)$, streptomycin $(84.44 \%)$, nalidixic acid $(77.78 \%)$, sulphafurazole (64.44\%), ampicillin (62.22\%), chloramphenicol $(46.67 \%)$ and least resistance was found against ciprofloxacin $(31.11 \%)$. Four penta MDR (ACSSuT) and two tetra MDR (ASSuT) resistance patterns were found S. Typhimurium strains. In S. Enteritidis strains, a high level of resistance was found against sulphonamides and streptomycin $(92.31 \%)$ with the least resistance against ciprofloxacin (11.54\%). Two penta MDR (ACSSuT) and six tetra MDR (ASSuT) resistance patterns were found. The presence of high antimicrobial resistance in zoonotic S. Typhimurium and Enteritidis in the broiler meat production chain is alarming. Immediate action and appropriate measures are required to control over the counter and irrational use of antibiotics both in poultry and humans. (C) 2021 Friends Science Publishers
\end{abstract}

Keywords: Salmonella Typhimurium; Salmonella Enteritidis; Antimicrobial resistance; Punjab

\section{Introduction}

Non-typhoidal Salmonella is one of the important zoonotic pathogens affecting both humans and animals is also considered the leading cause of bacterial diarrhea in the entire world. An estimated 153 million cases and 57000 deaths of nontyphoidal human salmonellosis are reported every year worldwide (Brunette 2017; Sharma et al. 2019). The majority of human cases of non-typhoidal Salmonella are foodborne. Infection caused by non-typhoidal Salmonella is usually self-limiting and in most cases, antimicrobial treatment is not required.(Angulo et al. 2000). However antimicrobial resistance is relevant in 3-10\% of cases where the infection is caused by invasive strains resulting in bacteremia and life-threatening conditions particularly in young and immunocompromised individuals
(Okeke et al. 2005; Chen et al. 2013).

The rise of antimicrobial resistance is a growing public health issue worldwide due to extensive use in agriculture, food and veterinary medicine which need to be controlled at the international level (De Oliveira et al. 2005). The development of Multi-Drug Resistance among foodborne pathogens is increasing dramatically all over the world and among these AMR in Salmonella is a serious emerging issue (Bronzwaer et al. 2002; Chen et al. 2013; Eng et al. 2015). Zoonotic non-typhoidal Salmonella has the properties of acquiring antimicrobial resistance over the years (Michael and Schwarz 2016). However the rate of antimicrobial resistance varies with different antibiotics and serotypes and Salmonella enterica serovar Enteritidis followed by Salmonella Typhimurium is relatively more susceptible to antimicrobial resistance than others, is one of

To cite this paper: Sadiq S, M Ahmad, M Chaudhry, H Akbar, MH Mushtaq, J Sadiq, S Hasan (2021). Phenotypic antimicrobial resistance patterns in Salmonella typhimurium and Enteritidis strains isolated from human, food, and environmental samples of broiler meat production chain in Punjab. Intl J Agric Biol 26:263-269 
the most prevalent serotypes ( $\mathrm{Su}$ et al 2004; Chen et al. 2013). A much higher rate of antibiotic resistance is also found in, another important serovar prevalent worldwide Centers for Disease Control and Prevention consider MDR Salmonella a serious public health threat that should be controlled efficiently on an urgent basis to avoid aggravated circumstances. Conventional antimicrobial agents such as sulphamethoxazole, chloramphenicol, ampicillin and trimethoprim are used as traditional first-line treatments for treating Salmonella. Salmonella enterica spp which are resistant to conventional antimicrobials are denoted as multidrug-resistant (MDR) Salmonella (Su et al. 2004; Eng et al. 2015; Bugarel et al. 2017). Resistance to the secondline treatment including fluoroquinolones which were used as a choice of treatment in MDR regions has also been reported (Chau et al. 2007; Klemm et al. 2018). Antimicrobial resistance both in typhoidal and nontyphoidal Salmonella is increasing in developing countries particularly the Indian subcontinent and Southeast Asia due to extensive use of antibiotics in public hospitals and communities (Threlfall 2002; Sharma et al. 2019). This situation is even grimmer in middle and low-income countries like Pakistan where there is no law enforcement to control the overwhelming sale of antibiotics. In Pakistan like our neighboring country, antibiotics are easily available in pharmacies and anyone can purchase over the counter without the prescription of health practitioners. (Sharma et al. 2019)

Until now in Pakistan, limited data is available on the prevalence of zoonotic nontyphoidal Salmonella and its susceptibility to different antimicrobial agents as compared to typhoidal Salmonella. A few studies have been reported on antimicrobial resistance of Salmonella Enteritidis and Salmonella Typhimurium in regions including Faisalabad (Akhtar et al. 2010; Wajid et al. 2018), in Sawat (Uddin et al. 2018) and Karachi (Shah and Korejo 2012).

The objective of this study was to check antimicrobial resistance in Salmonella Enteritidis and Salmonella Typhimurium isolated from different sources of the poultry food chain and humans in Lahore.

\section{Materials and Methods}

A cross-sectional study was conducted from 2017-2019 and samples were collected from different steps of the broiler meat production chain. Isolates from different sources including human, food and, the environment were used For the present study, a total of 71 Salmonella strains (Salmonella Typhimurium, $\mathrm{n}=45$; Salmonella Enteritidis, $\mathrm{n}$ $=26)$ were included for antimicrobial susceptibility testing (Table 1).

All Salmonella isolates were initially confirmed by biochemical tests. Salmonella serovar Typhimurium and Enteritidis and were confirmed through PCR by targeting their specific spy and, $s d f$ genes, respectively (Alvarez et al. 2004).

\section{Antimicrobial susceptibility testing by disc diffusion method}

Antimicrobial susceptibility testing was performed by Kirby-Beur disc diffusion method according to Clinical and Laboratory Standards Institute (CLSI) guidelines. The antibiotics which are commonly used in poultry production were selected and purchased. The antibiotics disks (OXOID, Thermo Scientific Ltd). and their concentration (microgram) used were, ampicillin 25 (AMP), amoxicillin 10 (AML), gentamicin $10(\mathrm{CN})$, streptomycin $10(\mathrm{~S})$, ciprofloxacin 5 (CIP), nalidixic acid 30 (NA), trimethoprim 5 (W), tetracycline 30 (TE), sulphafurazole 300 (SF) and chloramphenicol $30(\mathrm{C})$.

\section{Refreshing of isolates and preparation of inoculum}

Purified preserved cultures of Salmonella Typhimurium and Salmonella Enteritidis were refreshed in $10 \mathrm{~mL}$ tryptone soy broth with an overnight incubation at $37^{\circ} \mathrm{C}$. A loopful of the bacterial isolate was then streaked on nutrient agar plates and incubated for $24 \mathrm{~h}$ at $37^{\circ} \mathrm{C}$. Four to five well isolated and purified colonies were picked from plates with sterilized loop and properly mixed in test tubes containing $10 \mathrm{~mL}$ phosphate buffer saline. The turbidity of bacterial culture was standardized at $0.5 \mathrm{McF}$ arland (CLSI 2005). Another test tube containing $10 \mathrm{~mL}$ of PBS solution without inoculum was used as blank.

Approximately $20 \mathrm{~mL}$ of Mueller-Hinton agar (OXOID, Ltd) medium was poured into $90 \mathrm{~mm}$ diameter sterile Petri dishes to a depth of $4 \mathrm{~mm}$ with overnight incubation at $37^{\circ} \mathrm{C}$ to check for sterility (Kebede et al. 2016). After the adjustment of absorbance value, bacterial culture was spread on the Mueller-Hinton agar (OXOID, Ltd ) plate within 15 min with the help of a sterilized cotton swab. Briefly, the swab was dipped in a culture mixture and excess liquid was removed by squeezing the swab on the walls of the test tube. The swab was then streaked over the entire surface of the agar three times by rotating the plate approximately $60^{\circ}$ after each application to ensure an even distribution of the inoculum. The plates were then allowed to dry for $5 \mathrm{~min}$ before the application of antibiotic discs. A total of five discs were applied on a single plate for accurate measurement of the zones. The zones were measured with a calibrated scale according to Clinical \& Laboratory Standards Institute (CLSI) guidelines.

\section{Results}

The zones of inhibitions were measured according to the CLSI guidelines as resistant and sensitive. Fig. 1 summarizes the resistance of all S. Typhimurium serovars to 10 antimicrobial agents (Fig. 1).

Table 2 shows the antimicrobial resistance percentages of different antimicrobials against $S$. Typhimurium isolated from human, food and environmental samples. All the 
Phenotypic Antimicrobial Resistance in S. Typhimurium and Enteritidis / Intl J Agric Biol, Vol 26, No 2, 2021

Table 1: Salmonella Typhimurium and Enteritidis isolated from different sources

\begin{tabular}{lllll}
\hline & Type of sample & Salmonella Typhimurium & Salmonella Enteritidis & Total \\
\hline Humans & Hand swab & 7 & 2 & 9 \\
& Stool samples & 2 & 0 & 2 \\
Food samples & Chicken meat sample & 10 & 4 & 14 \\
Environmental samples & Egg samples & 7 & 4 & 16 \\
& Commercial broiler farms & 6 & 4 & 10 \\
Total & Transportation van samples & 13 & 6 & 1 \\
\hline
\end{tabular}

Table 2: Antimicrobial resistance (\%) in Salmonella Typhimurium isolated from different sources

\begin{tabular}{|c|c|c|c|c|c|c|c|c|c|c|c|}
\hline Sample Type (Typhimurium) & No. of isolates tested & AMP & AML & $\mathrm{S}$ & SF & CIP & $\mathrm{C}$ & NA & $\mathrm{TE}$ & $\mathrm{CN}$ & $\mathrm{W}$ \\
\hline Human samples & 9 & 88.89 & 100 & 77.78 & 88.89 & 44.44 & 11.11 & 66.67 & 88.89 & 100 & 88.89 \\
\hline Food samples & 17 & 23.53 & 94.12 & 76.47 & 35.29 & 29.41 & 29.41 & 64.71 & 100 & 76.47 & 88.24 \\
\hline
\end{tabular}

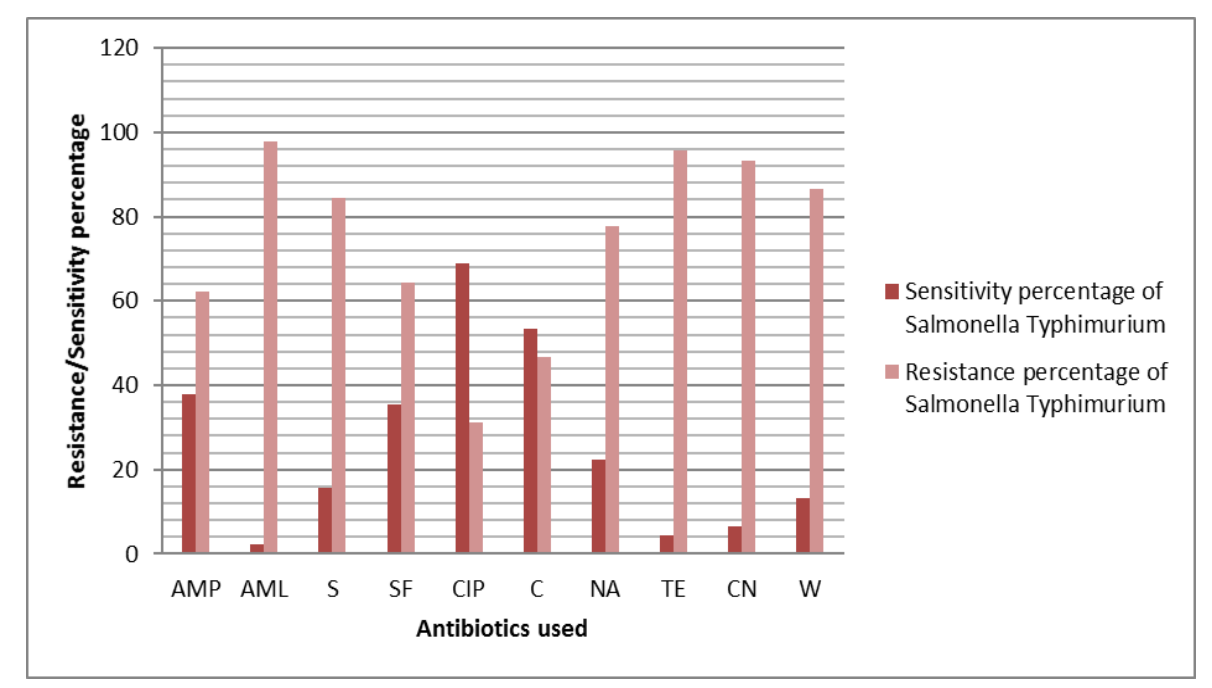

Fig. 1: Resistance (\%) of Salmonella Typhimurium isolated from different sources against 10 antibiotics

strains $(100 \%)$ were resistant to at least one antimicrobial agent. The highest level of resistance was found in amoxicillin (AML), gentamycin $(\mathrm{CN})$, tetracycline (TE), and, trimethoprim (W). All (100\%) of human and environmental samples while $(94.12 \%)$ of food samples were resistant to AML. Similarly, (100\%) of human and environmental samples and $(76.64 \%)$ were resistant to gentamycin $(\mathrm{CN})$. The higher level of resistance with $(100 \%)$ of food samples, $(94.74 \%)$ of environmental samples and, $(88.89 \%)$ of human samples were resistant to tetracycline (TE). An equal level of high resistance was found $(88 \%)$ in human food and environmental samples. A moderate level of resistance was found against AMP, SF, S, NA, C and CIP (in declining order) (Table 2).

A total of 25 resistance patterns were found (Table 3) with the most common (16\%) resistant pattern was (AMP, AML, S, SF, C, NA, TE, CN, W). According to the patterns of antimicrobial resistance, the highest resistance was shown in Salmonella Typhimurium strains isolated from vehicles $(13.33 \%)$ followed by chicken samples $(28.8 \%)$, human samples (20\%), egg samples $(15.5 \%)$ and (13.3\%) in broiler farms samples. Four penta MDR (ACSSuT) and two tetra MDR (ASSuT) pattern were found is S. Typhimurium. (Table 3).

Fig. 2 showed the resistance percentages of Salmonella Enteritidis isolated from different sources against 10 antibiotics with the highest resistance found against sulphonamides and streptomycin. (Fig. 2).

A great proportion of antimicrobial resistance was found in Salmonella Enteritidis strains with (96\%) of the isolated strains showed resistance to at least one antimicrobial agent (Table 4). The highest level of resistance was found in streptomycin (S), tetracycline (TE), nalidixic acid (NA) and CN. All the human and environmental $(100 \%)$ samples while $(92.31 \%)$ of food samples were resistant to streptomycin (S). Similarly $(100 \%)$ of human samples, $(92.31 \%)$ of food samples and $(91 \%)$ of environmental samples were resistant to tetracycline (TE). Similarly, $(100 \%)$ of human and environmental samples and $(76.64 \%)$ were resistant to gentamicin (CN). Again (100\%) of human samples, $(69.23 \%)$ and $(61.54 \%)$ of food samples, (36\%) and (64\%) 
Sadiq et al. / Intl J Agric Biol, Vol 26, No 2, 2021

Table 3: Antimicrobial resistance Patterns of Salmonella Typhimurium

\begin{tabular}{|c|c|c|c|c|c|c|c|}
\hline Antibiotics pattern & Human samples & Chicken samples & Egg samples & Vehicle samples & Broiler farm samples & Total & Percentage \\
\hline AMP,AML,S,CN & 0 & 0 & 0 & 0 & 1 & 1 & $2 \%$ \\
\hline AML,TE,CN,W & 0 & 0 & 1 & 0 & 0 & 1 & $2 \%$ \\
\hline AML,CIP,TE,W & 0 & 0 & 1 & 0 & 0 & 1 & $2 \%$ \\
\hline AML,S,SF,NA,TE, & 0 & 2 & & 0 & 0 & 2 & $4 \%$ \\
\hline AML,S,TE,CN,W & 0 & 0 & 1 & 0 & 0 & 1 & $2 \%$ \\
\hline AML,S,SF,TE,CN,W & 0 & 1 & 1 & 0 & 0 & 2 & $4 \%$ \\
\hline AMP,AML,S,CIP,TE,W & 0 & 0 & 1 & 0 & 0 & 1 & $2 \%$ \\
\hline AMP,AML,S,NA,TE,CN & 1 & 0 & 0 & 0 & 0 & 1 & $2 \%$ \\
\hline AML,S,C,NA,TE,CN,W & 0 & 1 & 1 & & 1 & 3 & $7 \%$ \\
\hline AMP,AML,C,NA,TE,CN,W & 0 & 0 & 0 & 1 & 0 & 1 & $2 \%$ \\
\hline AMP,AML,S,SF,NA,TE,CN & 0 & 1 & 0 & 0 & 0 & 1 & $2 \%$ \\
\hline AMP,AML,SF,NA,TE,CN,W & 1 & 0 & 0 & 0 & 0 & 1 & $2 \%$ \\
\hline AMP,AML,S,NA,TE,CN,W & 0 & 2 & 0 & 0 & 0 & 2 & $4 \%$ \\
\hline AMP,AML,S,SF,CIP,NA,TE,CN & 1 & 0 & 0 & 1 & 0 & 2 & $4 \%$ \\
\hline AMP,AML,SF,C,NA,TE,CN,W & 1 & 0 & 0 & 1 & 0 & 2 & $4 \%$ \\
\hline AML,S,CIP,C,NA,TE,CN,W & 0 & 1 & 0 & 0 & 0 & 1 & $2 \%$ \\
\hline AMP,AML,S,C,NA,TE,CN,W & 0 & 0 & 0 & 2 & 0 & 2 & $4 \%$ \\
\hline AML,S,SF,C,NA,TE,CN,W & 0 & 0 & 0 & 0 & 1 & 1 & $2 \%$ \\
\hline S,SF,CIP,C,NA,TE,CN,W & 0 & 0 & 0 & 0 & 0 & 0 & $0 \%$ \\
\hline AMP,AML,S,SF,NA,TE,CN,W & 1 & 1 & 0 & 0 & 1 & 3 & $7 \%$ \\
\hline AMP,AML,CIP,C,NA,TE,CN,W & 0 & 1 & 0 & 0 & 0 & 1 & $2 \%$ \\
\hline AML,S,SF,CIP,C,NA,TE,CN,W & 1 & 0 & 1 & 0 & 2 & 4 & $9 \%$ \\
\hline AMP,AML,S,SF,C,NA,TE,CN,W & 1 & 0 & 0 & 6 & 0 & 7 & $16 \%$ \\
\hline AMP,AML,S,SF,CIP,NA,TE,CN,W & 2 & 0 & 0 & 1 & 0 & 3 & $7 \%$ \\
\hline \multirow[t]{2}{*}{ AMP,AML,S,SF,CIP,C,NA,TE, CN,W } & 0 & 0 & 0 & 1 & 0 & 1 & $2 \%$ \\
\hline & 9 & 0 & 1 & 8 & 2 & 45 & $100 \%$ \\
\hline
\end{tabular}

Table 4: Antimicrobial resistance (\%) in Salmonella Enteritidis isolated from different sources

\begin{tabular}{|c|c|c|c|c|c|c|c|c|c|c|c|}
\hline Samples (Enteritidis) & No. of isolates tested & AMP & AML & $\mathrm{S}$ & SF & CIP & $\mathrm{C}$ & NA & $\mathrm{TE}$ & $\mathrm{CN}$ & $\mathrm{W}$ \\
\hline Human samples & 2 & $50 \%$ & $50 \%$ & $100 \%$ & $50 \%$ & $50 \%$ & $50 \%$ & $100 \%$ & $100 \%$ & $100 \%$ & $50 \%$ \\
\hline Food samples & 13 & 53.85 & $62 \%$ & 92.31 & 61.54 & $0 \%$ & 23.08 & 61.54 & 92.31 & 69.23 & 46.15 \\
\hline Environmental samples & 11 & $45 \%$ & $45 \%$ & $100 \%$ & 63.64 & 9.09 & $45 \%$ & $64 \%$ & $91 \%$ & $36 \%$ & $45 \%$ \\
\hline
\end{tabular}

of environmental samples were resistant to gentamicin $(\mathrm{CN})$ and nalidixic acid (NA) respectively. A moderate level of resistance was found against AMP, AML, SF, W, C and CIP. (in declining order) (Table 4). A total of 22 resistance patterns were found for Salmonella Enteritidis with the most common pattern among all samples were $(\mathrm{S}, \mathrm{NA}, \mathrm{AML}, \mathrm{W}$, TE, SF, AMP) which was $16 \%$ (Table 5). According to the patterns of antimicrobial resistance highest resistance was shown in S. Enteritidis strains isolated from egg samples (34.6\%) followed by transportation van samples $(23.0 \%)$, chicken samples and broiler farms samples (15.3\%), human samples (7.6\%) and least in chopping board samples (3.6\%). Two penta MDR (ACSSuT) and six tetra MDR (ASSuT) resistance patterns were found in S. Enteritidis (Table 5).

\section{Discussion}

In developing countries of Asia including Pakistan antimicrobial drugs are extensively used in food-producing animals and poultry for growth promotion and prophylaxis. Food-borne bacteria can attain resistance as a result of extensive use in food animals and can transmit these resistance genes to humans via the food chain (Bouchrif $e t$ al. 2009). The importance of antimicrobial resistance against zoonotic Salmonella serovar Enteritidis and
Typhimurium has been shown in this study. In our study, exponentially high resistance was found in Salmonella Typhimurium isolates where all $(100 \%)$ of the strains isolated from different sources were resistant to at least one antibiotic. One study from Minnesota reported (89\%) of Salmonella Typhimurium isolated from animals and (44\%) from humans were resistant to at least one antibiotic (Wedel et al. 2005). In two studies from Italy, one reported (75\%) of S. Typhimurium were resistant to at least one antimicrobial agent (Busani et al. 2004), and the second reported (87\%) of human isolates and $(81 \%)$ from animal source showed resistance to at least one antibiotic (Graziani et al. 2008) The high level of resistance found in our study may be due to widespread and over the counter use of antimicrobials in humans and veterinary (poultry) medicine in Pakistan.

According to the source of isolates a high level of resistance was found (76.64-100\%) among isolates of humans, environmental and, food against amoxicillin (94-100\%), gentamicin (76.64-100\%), tetracycline $(88.89 \%)$ and trimethoprim (88\%). Moderate level of resistance was found against (in declining order) ampicillin (45-50\%), sulphonamides (35-88\%), Streptomycin (76-89\%), nalidixic acid (64-94\%), chloramphenicol $(11-73 \%)$ and ciprofloxacin $(26-44 \%)$. Our results are inconsistent with reports from Italy that showed resistance 
Table 5: Anti Microbial Resistance Patterns of Salmonella Enteritidis

\begin{tabular}{|c|c|c|c|c|c|c|c|}
\hline AMR Pattern & Human samples & chicken samples & egg samples & transportation van samples & broiler farm samples & Chopping board & Total \\
\hline $\mathrm{C}, \mathrm{S}$ & 0 & 0 & $1(11 \%)$ & 0 & 0 & 0 & $1(4 \%)$ \\
\hline $\mathrm{S}, \mathrm{TE}$ & 0 & $1(25 \%)$ & $1(11 \%)$ & 0 & 0 & 0 & $2(8 \%)$ \\
\hline $\mathrm{CN}, \mathrm{S}, \mathrm{TE}$ & 0 & 0 & 0 & 0 & $1(25 \%)$ & 0 & $1(4 \%)$ \\
\hline S,NA,TE,AMP & 0 & 0 & $1(11 \%)$ & 0 & 0 & 0 & $1(4 \%)$ \\
\hline $\mathrm{S}, \mathrm{CIP}, \mathrm{W}, \mathrm{SF}, \mathrm{C}$ & 0 & 0 & 0 & 0 & $1(25 \%)$ & 0 & $1(4 \%)$ \\
\hline CN,S,TE,SF,AMP & 0 & 0 & 0 & $1(17 \%)$ & 0 & 0 & $1(4 \%)$ \\
\hline CN,NA,AML,W,TE & 0 & $1(25 \%)$ & 0 & 0 & 0 & 0 & $1(4 \%)$ \\
\hline CN,S,NA,AML,TE & 0 & 0 & 0 & $1(17 \%)$ & 0 & 0 & $1(4 \%)$ \\
\hline S,NA,TE,SF,AMP,C & 0 & 0 & 0 & $1(17 \%)$ & 0 & 0 & $1(4 \%)$ \\
\hline S,NA,W,TE,SF,C & 0 & 0 & 0 & $1(17 \%)$ & 0 & 0 & $1(4 \%)$ \\
\hline CN,S,NA,AML,TE,SF & 0 & 0 & 0 & $1(17 \%)$ & 0 & 0 & $1(4 \%)$ \\
\hline CN,S,CIP,NA,W,TE & $1(50 \%)$ & 0 & 0 & 0 & 0 & 0 & $1(4 \%)$ \\
\hline CN,S,NA,TE,SF,C & 0 & $1(25 \%)$ & 0 & 0 & 0 & 0 & $1(4 \%)$ \\
\hline CN,S,AML,W,TE,SF & 0 & 0 & $1(11 \%)$ & 0 & 0 & 0 & $1(4 \%)$ \\
\hline CN,S,NA,AML,TE,SF,AMP & 0 & 0 & $1(11 \%)$ & 0 & 0 & 0 & $1(4 \%)$ \\
\hline S,NA,AML,W,TE,SF,AMP & 0 & 0 & $1(11 \%)$ & 0 & $2(50 \%)$ & $1(100 \%)$ & $4(16 \%)$ \\
\hline S,NA,AML,W,TE,AMP,C & 0 & 0 & 0 & $1(17 \%)$ & 0 & 0 & $1(4 \%)$ \\
\hline S,NA,AML,W,TE,SF,C & 0 & $1(25 \%)$ & 0 & 0 & 0 & 0 & $1(4 \%)$ \\
\hline CN,S,AML,W,TE,SF,AMP & 0 & 0 & $1(11 \%)$ & 0 & 0 & 0 & $1(4 \%)$ \\
\hline CN,S,NA,AML,AMP,TE,SF,C & $1(50 \%)$ & 0 & 0 & 0 & 0 & 0 & $1(4 \%)$ \\
\hline CN,S,NA,AML,TE,SF,W,AMP & 0 & 0 & $1(11 \%)$ & 0 & 0 & 0 & $1(4 \%)$ \\
\hline S,NA,AML.W,TE,AMP,SF,CN & 0 & 0 & $1(11 \%)$ & 0 & 0 & 0 & $1(4 \%)$ \\
\hline & 2 & 4 & 9 & 6 & 4 & 1 & 26 \\
\hline
\end{tabular}

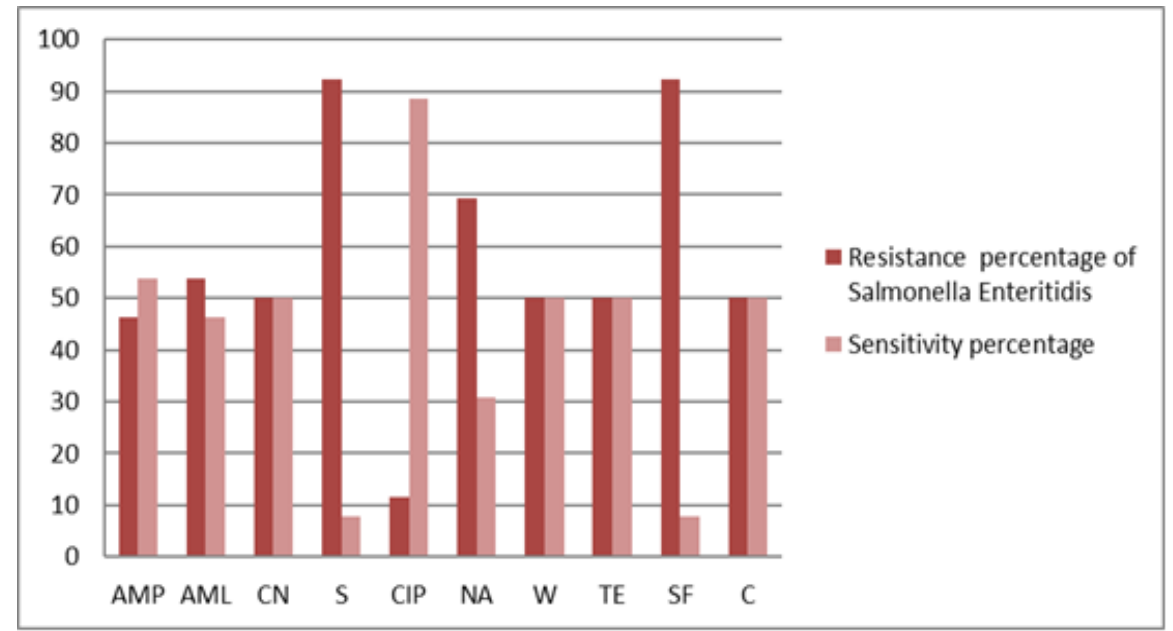

Fig. 2: Resistance of Salmonella Enteritidis isolated from different sources

to ampicillin $(67.6 \%)$, tetracycline $(73.6 \%)$, streptomycin (65.4\%), sulfonamides $(73.3 \%)$ and chloramphenicol $(32.3 \%)$ (Graziani et al. 2008). Another study reported from the UK showed (82\%) of S. Typhimurium were predominately resistant to, sulfonamides, streptomycin, tetracycline's, ampicillin, and chloramphenicol, while (20\%) were resistant to trimethoprim (Threlfall et al. 2003). Our results are also nearly equal to those reported from Italy, showed (83.9\%) of S. Typhimurium isolates showed resistance to tetracycline, sulfamethoxazole, streptomycin, chloramphenicol and ampicillin (De Vito et al. 2015). Another study from Ireland reported $(77.6 \%)$ of Typhimurium isolates were resistant to ampicillin, chloramphenicol, streptomycin, sulfonamides and tetracycline (Gorman and Adley 2004). In the present study, a very low level of resistance was found against ciprofloxacin as also reported from Italy where all $S$. typhiurium isolates were susceptible to ciprofloxacin (Busani et al. 2004).

A total of 26 resistance patterns were found in Salmonella Typhimurium isolates of which 4 (ACSSuT) and 2 (ASSuT) patterns were found. The highest prevalent pattern was ((ASSuT) AML, NA, CN, W) which was present in $16 \%$ of MDR Typhimurium isolates. Our results are inconsistent with reports from China, Italy where the most frequently observed patterns of resistance were (ACSSuT) and (ASSuT) (Graziani et al. 2008; Wang et al. 2019).

A great proportion of antimicrobial resistance was also found in S. Enteritidis strains with, (96\%) of the isolated 
strains showed resistance to at least one antimicrobial agent. Maximum level $(92.31 \%)$ of resistance was shown against sulphonamides and streptomycin and $(69.23 \%)$ for nalidixic acid. Our results are supported by a study in Brazil where $(91 \%)$ of $S$. Enteritidis showed resistance to at least one antimicrobial agent with the maximum level of resistance was found against sulphonamides (75.8\%) (Dias de Oliveira et al. 2005). One study from Korea reported that (90\%) of $S$. Enteritidis strains were resistant to sulphonamides and nalidixic acid (Hur et al. 2011) which is very close to our study. Another study from Taiwan reported (70\%) resistance to streptomycin and (75\%) to tetracyclin in two studies from Brazil reported (73.3\%) and (28.12\%) of S. enetritidis were resistant to nalidixic acid (Chu et al. 2009; Campioni et al 2012; Campioni et al. 2014). Nalidixic acid that targets DNA gyrase is one of the common antimicrobials used for the treatment of salmonellosis.

In the present study moderate level of resistance was found against ampicillin (46.2\%), amoxicillin (53.8\%), gentamicin $(50 \%)$, trimethoprim $(50 \%)$, tetracycline $(50 \%)$, and chloramphenicol (50\%). Lower level resistance was shown from a study in Brazil where a low level of resistance was found for tetracycline (15.4\%), streptomycin $(7.7 \%)$, gentamicin $(5.5 \%)$, trimethoprim $(3.3 \%)$, ampicillin $(1.1 \%)$, and chloramphenicol (1.1\%). One study from Iran in agreement with our study reported $(100 \%)$ resistance of S. Enteritidis against ampicillin (70\%) against streptomycin and $60 \%$ against gentamicin(Ghazaey and Mirmomeni 2012). The high level of resistance against different antibiotics may be due to without prescription over the counter use in human and veterinary medicine.

In the present study, a total of 22 resistance patterns were found and the most prevalent pattern (16\%) was ((ASSuT) W, AML, NA). A total of two (ACSSuT) and four (ASSuT) were found in S.Enteritidis isolates. Our results are in agreement with the study reported from Spain where 23 different resistant patterns were found in $S$. Enteritidis (Carramiñana et al. 2004).

\section{Conclusion}

An alarmingly high level of resistance was found in both Salmonella Typhimurium and Enteritidis isolated from humans, food and environmental sample. In addition to this, our study isolates were resistant to sulphonamides, nalidixic acid, ampicillin, streptomycin and tetracycline is the indication of extensive use of these antibiotics in human and veterinary medicine. In our study low level of resistance and a high level of susceptibility were found against ciprofloxacin. So fluoroquinolones can be the choice for treating salmonellosis in our poultry industry.

\section{Acknowledgments}

Authors acknowledge the poultry farmers and retail shop owners for provision of samples. Authors are also thankful to the Laboratory staff of Department of Epidemiology and Public Health for assistance in conduct of current study. The current research study was funded under HEC Indigenous $\mathrm{PhD}$ program.

\section{Author Contributions}

MDA, MC and HA planned the study, SS conduct the whole research work and MHM, JS and SH help in formatting of manuscript.

\section{Conflicts of Interest}

Authors declare no conflict of interest.

\section{Data Availability}

Data are available from the first author on reasonable request.

\section{Ethics Approval}

Not applicable in this paper

\section{References}

Akhtar F, I Hussain, A Khan, SU Rahman (2010). Prevalence and antibiogram studies of Salmonella enteritidis isolated from human and poultry sources. Pak Vet J 30:25-28

Alvarez J, M Sota, AB Vivanco, I Perales, R Cisterna, A Rementeria, J Garaizar (2004). Development of a multiplex PCR technique for detection and epidemiological typing of Salmonella in human clinical samples. J Clin Microbiol 42:1734-1738

Angulo FJ, KR Johnson, RV Tauxe, ML Cohen (2000). Origins and consequences of antimicrobial-resistant nontyphoidal Salmonella: Implications for the use of fluoroquinolones in food animals. Microb Drug Resist 6:77-83

Bouchrif B, P Bianca, M Murgia, A Piana, N Cohen, MM Ennaji, S Rubino M Timinouni (2009). Prevalence and antibiotic-resistanceof Salmonella isolated from food in Morocco. J Infect Dev Ctries 3:35-40

Bronzwaer SLAM, OBU Cars, S Mölstad, W Goettsch, IK Veldhuijzen, JL Kool, MJW Sprenger, JE Degener, European Antimicrobial Resistance Surveillance S. 2002. A European study on the relationship between antimicrobial use and antimicrobial resistance. Emerg Infect Dis 8:278-282.

Brunette GW (2017). CDC Yellow Book 2018: Health Information for International Travel. Oxford University Press, Oxford, UK

Bugarel M, A Tudor, GH Loneragan, KK Nightingale (2017). Molecular detection assay of five Salmonella serotypes of public interest: Typhimurium, Enteritidis, Newport, Heidelberg, and Hadar. $J$ Microbiol Meth 134:14-20

Busani L, C Graziani, A Battisti, A Franco, A Ricci, D Vio, E Digiannatale, F Paterlini, M D'Incau, S Owczarek, A Caprioli, I Luzzi (2004). Antibiotic resistance in Salmonella enterica serotypes Typhimurium, Enteritidis and Infantis from human infections, foodstuffs and farm animals in Italy. Epidemiol Infect 132:245-251

Campioni F, AMM Bergamini, JP Falcão (2012). Genetic diversity, virulence genes and antimicrobial resistance of Salmonella Enteritidis isolated from food and humans over a 24-year period in Brazil. Food Microbiol 32:254-264

Campioni F, MM Zoldan, JP Falcão (2014). Characterization of Salmonella Enteritidis strains isolated from poultry and farm environments in Brazil. Epidemiol Infect 142:1403-1410 
Carramiñana JJ, C Rota, I Agustín, A Herrera (2004). High prevalence of multiple resistance to antibiotics in Salmonella serovars isolated from a poultry slaughterhouse in Spain. Vet Microbiol 104:133-139

Chau TT, JI Campbell, CM Galindo, N Van Minh Hoang, TS Diep, TTT Nga, N Van Vinh Chau, PQ Tuan, AL Page, RL Ochiai, C Schultsz, J Wain, ZA Bhutta, CM Parry, SK Bhattacharya, S Dutta, M Agtini, B Dong, Y Honghui, DD Anh, DG Canh, A Naheed, MJ Albert, R Phetsouvanh, PN Newton, B Basnyat, A Arjyal, TTP La, NN Rang, LT Phuong, P Van Be Bay, L von Seidlein, G Dougan, JD Clemens, H Vinh, TT Hien, NT Chinh, CJ Acosta, J Farrar, C Dolecek (2007). Antimicrobial drug resistance of Salmonella enterica serovar typhi in Asia and molecular mechanism of reduced susceptibility to the fluoroquinolones. Antimicrob Agents Chemother 51:4315-4323

Chen HM, Y Wang, LH Su, CH Chiu (2013). Nontyphoid Salmonella infection: Microbiology, clinical features, and antimicrobial therapy. Pediatr Neonatol 54:147-152

Chu C, DW Wong, MH Wang, HH Lin, YS Chen, N Tien, MC Shih, TH Chen, CH Chiu (2009). Genotyping, plasmid analysis, and antimicrobial susceptibility of Salmonella enterica serotype enteritidis isolates from humans and chickens in central Taiwan. $J$ Formos Med Assoc 108:765-771

CLSI (2005). Performance standards for antimicrobial susceptibility testing fifteenth informational supplement M100-S15. In: Clinical and Laboratory Standards Institute, Wayne, Pennsylvania, USA

De Oliveira SID, FS Flores, LR dos Santos, A Brandelli (2005). Antimicrobial resistance in Salmonella enteritidis strains isolated from broiler carcasses, food, human and poultry-related samples. Intl J Food Microbiol 97:297-305

De Vito D, R Monno, F Nuccio, M Legretto, M Oliva, MF Coscia, AM Dionisi, C Calia, C Capolongo, C Pazzani (2015). Diffusion and persistence of multidrug resistant Salmonella typhimurium strains phage type DT120 in southern Italy. Biol Med Res Intl 2015; Article 265042

Dias de Oliveira Sl, F Siqueira Flores, LR dos Santos, A Brandelli (2005). Antimicrobial resistance in Salmonella enteritidis strains isolated from broiler carcasses, food, human and poultry-related samples. Intl J Food Microbiol 97:297-305

Eng SK, P Pusparajah, NS Ab Mutalib, HL Ser, KG Chan, LH Lee (2015). Salmonella: A review on pathogenesis, epidemiology and antibiotic resistance. Front Life Sci 8:284-293

Ghazaey S, MH Mirmomeni (2012). Microbial-resistant Salmonella enteritidis isolated from poultry samples. Rep Biochem Mol Biol $1: 9-13$

Gorman R, CC Adley (2004). Characterization of Salmonella enterica serotype typhimurium isolates from human, food, and animal sources in the Republic of Ireland. J Clin Microbiol 42:2314-2316

Graziani C, L Busani, A Dionisi, C Lucarelli, S Owczarek, A Ricci, M Mancin, A Caprioli, I Luzzi (2008). Antimicrobial resistance in Salmonella enterica serovar Typhimurium from human and animal sources in Italy. Vet Microbiol 128:414-418

Hur J, JH Kim, JH Park, YJ Le, JH Lee (2011). Molecular and virulence characteristics of multi-drug resistant Salmonella enteritidis strains isolated from poultry. Vet $J$ 189:306-311
Kebede A, J Kemal, H Alemayehu, S Habte Mariam (2016). Isolation, identification, and antibiotic susceptibility testing of Salmonella from slaughtered bovines and ovines in Addis Ababa Abattoir Enterprise, Ethiopia: A cross-sectional study. Intl J Bacteriol 2016; Article 3714785

Klemm EJ, S Shakoor, AJ Page, FN Qamar, K Judge, DK Saeed, VK Wong, TJ Dallman, S Nair, S Baker, G Shaheen, S Qureshi, MT Yousafzai, MK Saleem, Z Hasan, G Dougan, R Hasan (2018). Emergence of an Extensively Drug-Resistant Salmonella enterica serovar typhi clone harboring a promiscuous plasmid encoding resistance to fluoroquinolones and third-generation cephalosporins. mBio 9 :105-118

Michael GB, S Schwarz (2016). Antimicrobial resistance in zoonotic nontyphoidal Salmonella: An alarming trend? Clin Microbiol Infect 22:968-974

Okeke IN, R Laxminarayan, ZA Bhutta, AG Duse, P Jenkins, TF O'Brien, A Pablos-Mendez, KP Klugman (2005). Antimicrobial resistance in developing countries. Part I: Recent trends and current status. Lancet Infect Dis 5:481-493

Shah AH, NA Korejo (2012). Antimicrobial resistance profile of Salmonella serovars isolated from chicken meat. J Vet Anim Sci 2:40-46

Sharma J, D Kumar, S Hussain, A Pathak, M Shukla, V Prasanna Kumar, PN Anisha, R Rautela, AK Upadhyay, SP Singh (2019). Prevalence, antimicrobial resistance and virulence genes characterization of nontyphoidal Salmonella isolated from retail chicken meat shops in Northern India. J Food Cont 102: 104-111

Su LH, CH Chiu, C Chu, JT Ou (2004). Antimicrobial Resistance in Nontyphoid Salmonella Serotypes: A Global Challenge. Clin Infect Dis 39:546-551

Threlfall E, C Teale, R Davies, L Ward, J Skinner, A Graham, C Cassar, K Speed (2003). A comparison of antimicrobial susceptibilities in nontyphoidal Salmonellas from humans and food animals in England and Wales in 2000. Microb Drug Resist 9:183-189

Threlfall EJ (2002). Antimicrobial drug resistance in Salmonella: Problems and perspectives in food-and water-borne infections. FEMS Microbiol Rev 26:141-148

Uddin MN, MM Farooq, M Waqas, NU Khan, WA Khan, I Khan, N Karim, M Rizwan (2018). Antibiotic assays of Salmonella isolated from poultry chicken of various locations in districts Swat. Pure Appl Biol 7:78-84

Wajid M, AB Awan, MK Saleemi, J Weinreich, P Schierack, Y Sarwar, A Ali (2018). Multiple drug resistance and virulence profiling of Salmonella enterica serovars typhimurium and enteritidis from poultry farms of Faisalabad, Pakistan. Microb Drug Resist 25:133-142

Wang X, S Biswas, N Paudyal, H Pan, X Li, W Fang, M Yue (2019). Antibiotic resistance in Salmonella ryphimurium isolates recovered from the food chain through national antimicrobial resistance monitoring system between 1996 and 2016. Front Microbiol 10; Article 985

Wedel SD, JB Bender, FT Leano, DJ Boxrud, C Hedberg, KE Smith (2005). Antimicrobial-drug susceptibility of human and animal Salmonella typhimurium, Minnesota, 1997-2003. Emerg Infect Dis 11:1899-1906 